\title{
Diagnostics Development for Quasi-Steady-State Operation of the Wendelstein 7-X Stellarator ${ }^{\mathrm{a})}$
}

\author{
R. König ${ }^{1, b)}$, J. Baldzuhn ${ }^{1}$, C. Biedermann ${ }^{1}$, R. Burhenn ${ }^{1}$, S. Bozhenkov ${ }^{1}$, A. \\ Cardella ${ }^{1}$, M. Endler ${ }^{1}$, H.-J. Hartfuss ${ }^{1}$, D. Hathiramani ${ }^{1}$, D. Hildebrandt ${ }^{1}$, M.Hirsch ${ }^{1}$, \\ M. Jakubowski ${ }^{1}$, G. Kocsis ${ }^{2}$, P. Kornejev ${ }^{1}$, M. Krychowiak ${ }^{1}$, H. P. Laqua ${ }^{1}$, M. \\ Laux $^{1}$, J. W. Oosterbeek ${ }^{3}$, E. Pasch ${ }^{1}$, T. Richert ${ }^{1}$, W. Schneider ${ }^{1}$, T. Sunn- \\ Pedersen $^{1}$, H. Thomsen ${ }^{1}$, A. Weller ${ }^{1}$, A. Werner ${ }^{1}$, R. Wolf ${ }^{1}$, D. Zhang ${ }^{1}$, S. Zoletnik ${ }^{2}$ \\ ${ }^{1}$ Max-Planck-Institut für Plasmaphysik, EURATOM Association, D-17491, Greifswald, Germany \\ ${ }^{2}$ Wigner RCP, RMI, Association EURATOM, H-1121 Budapest, Konkoly Thege 29-33, Hungary \\ ${ }^{3}$ Eindhoven University of Technology, Den Dolech 2, 5612 AZ Eindhoven, The Netherlands
}

(Presented XXXXX; received XXXXX; accepted XXXXX; published online XXXXX)

The critical issues in the development of diagnostics which need to work robust and reliable under quasisteady state conditions for discharge durations of $30 \mathrm{~min}$ and which cannot be maintained throughout the one week duration of each operation phase of the Wendelstein 7-X stellarator are being discussed.

\section{INTRODUCTION}

In Greifswald, Germany, the superconducting advanced stellarator Wendelstein 7-X (W7-X) is presently being built. ${ }^{1}$ This device is designed for $10 \mathrm{MW}$ of ECRH heating power at $140 \mathrm{GHz}(\lambda=2.1 \mathrm{~mm})$ for up to 30 minutes duration twice per day. ${ }^{2}$ To minimize the wear and tear on the inter coil mechanical sliding support pads ${ }^{3}$ it is envisaged not to make any large changes to the magnetic configurations throughout the planned at least one week long operation periods. No manned access to the torus hall will be permitted with applied magnetic field, therefore, all diagnostics components need to be designed as robust as possible. Any electronics component which may fail needs to be kept outside the torus hall in order to remain accessible. Redundancy for critical items inside the torus hall as well as robustness of components is therefore of utmost importance and one of the driving guidelines in the diagnostics development process. After a commissioning phase of about one year, W7-X will start in the summer of 2015 with an uncooled divertor which will only be able to sustain $8 \mathrm{MW}$ of heating power for about 10 seconds. Nonetheless, nearly all diagnostics will already be designed for the quasi-continuous operation phase which is presently expected to start at the end of 2018. A completion phase of 15 month will be required before that date, during which the presently manufactured high heat load, actively cooled divertor will be installed. ${ }^{4}$ The concepts to be followed for quasi-continuously operating diagnostics are always quite different from the conventional approaches for short pulse devices. Very early on in the diagnostic design phase it became clear that starting off with simple only short pulse compatible designs, followed by a later hardening of diagnostics for quasicontinuous operation is just not feasible.,

In the following, the complexity of the various issues that need to be tackled in a large variety of ways is sketched out and examples of their implementation in a number of typical diagnostics are given.

\footnotetext{
a) Invited paper published as part of the Proceedings of the 19th Topical Conference on High-Temperature Plasma Diagnostics, Monterey, California, May, 2012

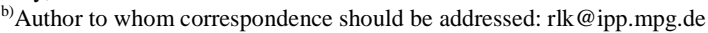

\section{COPING WITH THERMAL HEAT LOADS}

In quasi-continuously operating devices with pulse durations of up to 30 minutes almost all in-vessel components will reach their thermal equilibrium. In W7-X any component which is neither actively cooled nor thermally sufficiently well connected to cooled structures will easily reach radiative equilibrium temperatures of $500-800^{\circ} \mathrm{C}$ by exposure to plasma radiation alone. Any additional heat load, convective or induced by ECRH stray radiation, worsens the situation further. ${ }^{7}$ These three different kinds of heat sources require different measures to be taken to protect diagnostic components from their detrimental effects, which is why they are discussed separately in the following.

\section{A. Predominantly convective loads}

Predominantly convective loads are found on the divertor target tiles, which can handle up to $10 \mathrm{MW} / \mathrm{m}^{2}$, but are also expected where the plasma wall distance gets smaller than 100 $\mathrm{mm}$ (for some components even less than $60 \mathrm{~mm}$ ). Two types of carbon tiled water cooled heat shields ${ }^{8}$ are developed, covering about $40 \%$ of the W7-X wall, which can sustain average loads up to $150 \mathrm{~kW} / \mathrm{m}^{2}$ (respectively $350 \mathrm{~kW} / \mathrm{m}^{2}$ ) and locally up to 250 (500) $\mathrm{kW} / \mathrm{m}^{2}$. Additional diagnostic heat shields using the same technology have been specially designed to protect in-vessel diagnostics components like the diamagnetic loop and the SoftX-ray multi-camera tomography system (XMCTS) in the triangular shaped plasma plane. ${ }^{9}$ An efficient active cooling of all its components through a very careful thermo-mechanical design and small pinholes in the carbon tile structures were necessary in order to sustain the expected heat loads of up to $350 \mathrm{~kW} / \mathrm{m}^{2}$ (continuous load) onto the heat shield of the 20 in-vessel soft $\mathrm{x}$ ray cameras. ${ }^{10}$

\section{B. Predominantly plasma radiative loads}


Diagnostics components installed in the 117 diagnostic ports around the machine are usually either located at the very end of the typically 1.5 to $2 \mathrm{~m}$ long ports of W7-X, where plasma radiation induced thermal loads play no significant role anymore or they are at least installed so far retracted inside the ports that convection doesn't play any significant role. In this latter case the plasma facing diagnostics components need to sustain predominantly the heat load originating from plasma emission. Most of the power is radiated by the plasma at wavelengths $<6$ $\mathrm{nm} .{ }^{11}$ Except for very shallow angles of incidence, photons emitted at such short wavelengths are absorbed by the first component they hit. Therefore, expected heat load distributions across in-vessel components could be calculated with a Monte Carlo simulation code by simply launching photons from flux surfaces and counting their first collisions with surface primitives of in-vessel components. Such calculations were performed for different assumed plasma emission profiles, e.g. parabolic or high density detached divertor X-line radiation profiles. ${ }^{12}$ The calculations showed that heat loads of up to $80 \mathrm{~kW} / \mathrm{m}^{2}$ need to be expected at the location of the diagnostic port entrance surfaces. Water cooled stainless steel structures are sufficient to handle such loads. For example most of the planned visible and infrared plasma vessel and divertor imaging diagnostic components have been placed behind cooled SS plasma facing front plates. They are viewing the plasma only through a small pinhole which at the same time forms the entrance pupil of the observation optics. ${ }^{13}$ In cases where large observation windows were required, they were retracted as much as possible into the ports. Thereby the power loads are reduced to about $20-50 \mathrm{~kW} / \mathrm{m}^{2}$ in case of the Thomson Scattering $^{14}$ and CXRS observation systems. Various types of edge water cooled windows were specially designed for such conditions differing due to their different load requirements. ${ }^{15}$ In addition, these diagnostics are equipped with water cooled SS shutters.

\section{ECRH stray radiation loads}

The W7-X ECRH system can provide 10 MW of heating power for up to $30 \mathrm{~min}$. In X2 mode this radiation is well absorbed by the plasma for densities below the cut-off density of $1.2510^{20} \mathrm{~m}^{-3}$. However, for higher density operation in O2- (cutoff density $2.410^{20} \mathrm{~m}^{-3}$ ) and OXB-mode single path nonabsorbed power fractions are of the order of $20 \%$ and even higher values need to be expected near the cut-off densities. Allowing only a maximum of $1 \mathrm{MW}$ ECRH stray radiation this leads to levels of up to $80 \mathrm{~kW} / \mathrm{m}^{2}$ in the ECRH launching plane and about a factor 10 smaller values at the opposite side of the torus. With the absorption coefficients for steel and even carbon being only in the few \% range, this radiation usually undergoes a very large number of reflections and scatterings at the surface roughness until it is finally absorbed. Thus, the ECRH stray radiation behaves roughly like a collisionless gas spreading into vacuum. Simple model calculations with this assumption show that behind the first wall elements, deep inside smaller ports or even deep inside diagnostic components, the radiation level is often reduced by less than a factor 2-4 only. To ensure that diagnostic components can survive such high radiation loads often requires various special measures to be taken but also a good knowledge of the properties of the materials used for the diagnostics design. A special Microwave Stray Radiation Launch Facility (MISTRAL), which is basically a large Aluminum tank to which at one end the largest W7-X port flange has been attached, was built, to test the ECRH compatibility of various individual components and also the complete diagnostics. The radiation power from a single W7-X gyrotron is launched onto a mirror just inside the vessel which reflects the radiation along the wall thereby first spreading it like an annulus along the edge of the vessel wall. The roughness of the vessel wall surface gradually causes a diffuse reflection of the radiation also towards the central part of the chamber thereby creating a homogeneous stray radiation distribution within an about $80 \mathrm{~cm}$ diameter and $2 \mathrm{~m}$ long cylindrical area. Our largest components are being tested inside this homogeneous central region.

The experiments demonstrated that the thermal radiation emission coefficients of materials often determine as much as their ECRH absorption coefficients the final equilibrium temperature of components. The XMCTS diagnostic for example requires a number of flexible bellows for cables which can only sustain temperatures up to $250{ }^{\circ} \mathrm{C}$. It was found that SS bellows having high ECRH absorption ( 1-3\%) but also high thermal radiation emission coefficients after a few minutes already reach a too high equilibrium temperature of $320^{\circ} \mathrm{C}$ (Fig. 1). Coating the SS bellows with a $20 \mu \mathrm{m}$ copper layer significantly reduced the ECRH absorption $(<0.2 \%)$ and thus slowed down its heat-up significantly but the very low emission coefficient of $\mathrm{Cu}$ leads to an even higher equilibrium temperature which, however, over 30 minutes was not yet reached. However, the temperature after 30 min was still too high $\left(\sim 300{ }^{\circ} \mathrm{C}\right)$. Finally, a bronze bellows was used which has an ECRH absorption coefficient somewhere between $\mathrm{SS}$ and $\mathrm{Cu}$, a higher thermal emission coefficient and a better thermal conductivity than SS. The bronze bellows reached its equilibrium temperature of just below $250^{\circ} \mathrm{C}$ after 30 minutes.

In the design of diagnostics one needs to be particularly careful not to expose ceramic or insulating components to ECRH since most of these have extremely high ECRH absorption coefficients. This makes them act like a sink for ECRH stray radiation, due to the many reflections of the radiation before it is absorbed by the predominantly metal, low absorbing surfaces inside the plasma vessel. Therefore they very quickly heat up to unacceptably high temperatures if not well shielded or thermally well connected to cooled structures. The high absorption of some ceramics materials can on the other hand be exploited by coating cooled surfaces inside diagnostic cavities with special highly absorbing materials, like $\mathrm{B}_{4} \mathrm{C}^{16}$ or $\mathrm{Al}_{2} \mathrm{O}_{3} / \mathrm{TiO}_{2}$. These can significantly reduce the overall stray radiation level. A $150 \mu \mathrm{m}$ thick layer of the latter for example has an absorption coefficient at $140 \mathrm{GHz}$ of about $70 \% .{ }^{17}$ Another very efficient ECRH

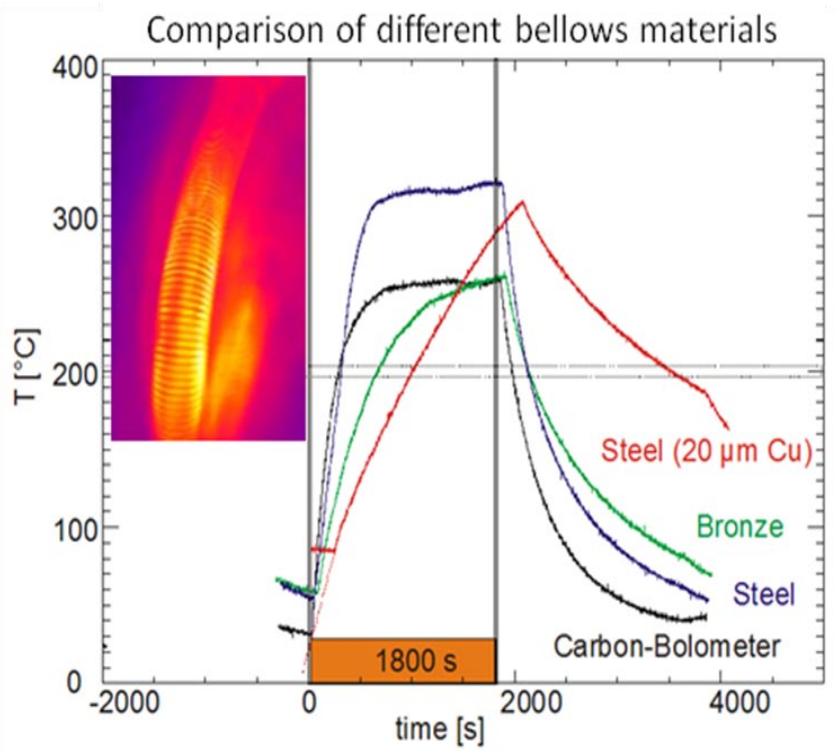

FIG. 1. Temperature evolution of various bellows exposed over $30 \mathrm{~min}$ to $40 \mathrm{~kW} / \mathrm{m}^{2}$ ECRH stray radiation 
radiation sink, which is used outside the vacuum vessel, is highly absorbing water flowing through a highly ECRH transparent Teflon ${ }^{\circledR}$ hose.

Flexible corrugated SS water cooling hoses with outer braiding, as used in the supply lines for the cooled wall protection components, were found to absorb significant stray radiation levels in their sub-wavelength SS mechanical support meshes. The thermal contact between the braiding and the cold corrugated hose inside is rather poor so that at the maximum stray radiation levels expected behind the first wall components of $40 \mathrm{~kW} / \mathrm{m}^{2}$ the temperature of the braiding still reached values of about $500^{\circ} \mathrm{C}$. This is already a temperature which may weaken the mechanical strength of the SS braiding. Further investigations of this issue are ongoing. Fortunately, the initially observed strong arcing across the meshes could in the meantime be attributed to the way the MISTRAL chamber is operated. To produce the relatively low stray radiation power levels of about $40 \mathrm{~kW} / \mathrm{m}^{2}$ with the 1MW ECRH gyrotrons, they need to be operated with a rather low duty cycle $(1: 20)$, i.e. with a series of rather short but very high power pulses leading to arcing. To prove the above point, a specially prepared experiment has been performed in which the power level launched into MISTRAL has been reduced to the actually required low continuously required value.

Exposing gaskets used in gas valves to ECRH stray radiation also showed that the O-rings FKM 70 Shore A green and ORAR00259-V70GA from the company Trelleborg absorbed about half of what black Viton ${ }^{\circledR}$ ones did. However, while the worst quality openly exposed gaskets were destroyed in $10 \mathrm{~s}$ none of the better quality so far failed in any of the gas valves installed on the MISTRAL chamber, showing that they are obviously sufficiently shielded within the vacuum valves from $\mathrm{VAT}^{\circledR}$ that we use. Also, window materials have different absorption coefficients $^{5}$ with diamond being most transparent and for example used as exit window for our $1 \mathrm{MW}$ output power gyrotrons. Sapphire is also known for its good transmission properties. However, one of our unprotected $\mathrm{ZnSe}$ infrared windows in an experiment once got heated up to temperatures above $400^{\circ} \mathrm{C}$ and became opaque in the visible. This is known to happen when it is being exposed to air at such high temperatures. This must have been caused by an unusually high power level since in all experiments in recent years no extra cooling of these windows was necessary.

ECRH shielding for diagnostic components can be provided by wire meshes or specially designed dichroic filters. ${ }^{18}$ To protect the neutral pressure measurement ionization gauges, which were affected by the stray radiation, dichroic filter plates made of 2 $\mathrm{mm}$ thick $\mathrm{Cu}$ discs with $0.7 \mathrm{~mm}$ diameter holes with $2 \mathrm{~mm}$ separations are being used. Our standard DN40CF flange disk with 337 holes reduces the molecular flow factor by a factor of 4.8. The $\mathrm{Cu}$ disc directly serves as the seal in the flange. Thin wire meshes placed in front of the infrared camera with its $\mathrm{ZnSe}$ lens not only heat up gradually, thereby increasing the background noise in IR camera images but are also the cause for significant ghosts/reflections across the entire image area even if only a small hot component is looked at. While no good protecting solution for the IR region has been found yet, in the visible spectral region a thin, as highly conductive as possible, transparent indium tin oxide (ITO) layer of about $1 \mu \mathrm{m}$ thickness can provide excellent ( $<0.5 \%$ transmission) ECRH shielding of camera detectors while still providing high transmission in the visible, as a sample produced for us by Melles Griot shows. ${ }^{7}$ Earlier papers show that with suitable production processes even visible transmission coefficients of up to $60 \%$ at $280 \mathrm{~nm}$ can be reached. ${ }^{19}$

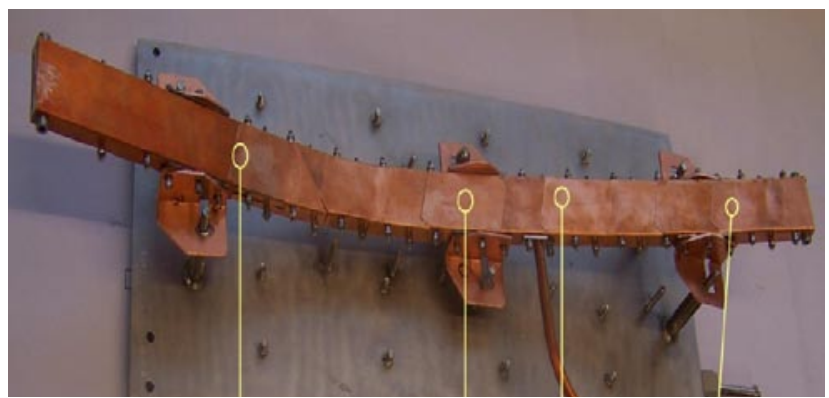

FIG. 2. Diamagnetic loop mock-up with full ECRH shielding

An ECRH stray radiation compatible electrical isolation flange has been designed. A $0.5 \mathrm{~mm}$ gap labyrinth, the surfaces of which we intend to coat with a $150 \mu \mathrm{m} \mathrm{Al}_{2} \mathrm{O}_{3} / \mathrm{TiO}_{2}$ absorber layer protects the isolating seal made of Peek ${ }^{\circledR}$ while the screws are insulated by Teflon ${ }^{\circledR}$ washers and tubes. This design has already been tested successfully in MISTRAL even without the absorber coating.

The magnetic diagnostics for $\mathrm{W} 7-\mathrm{X}$ require an enormously large effort to make them ECRH compatible. We have to ensure that the insulating material of the wires does not heat up to temperatures above $250{ }^{\circ} \mathrm{C}$. This means in case of the diamagnetic loops that the complete winding package needs to be tightly enclosed in a thin copper shield (Fig. 2). Initial simpler shielding solutions with hardly visible gaps resulted in overheating and destruction of the winding package of a mock-up tested in MISTRAL. Our initial intention had also been to invessel assemble the loop from a few sections inside our complex shaped plasma vessel by connecting the sections with specially designed multi-pin plugs. We learned, however, that it was not possible to keep the thermo-voltages at the connecting plug at acceptable levels. Our final solution became a specially produced continuous ribbon cable without any connections up to the flange at the air-vacuum barrier. The loop has now been equipped with five hinges such that it can be folded to fit through the W7-X manhole ports and can then be unfolded for assembly inside the machine. Of course the ECRH shield requires particular care in the hinge regions. The whole SS support structure for the ribbon cable being completely enclosed in copper protection sheets makes it necessary to ensure sufficient thermal anchoring to the cooled plasma vessel wall. As discussed above, here the poor thermal emission coefficient of $\mathrm{Cu}$ needs to be compensated by making use of its excellent thermal conductivity to transport the heat to the vessel wall.

The ECRH stray radiation compatible design of the continuous and segmented Rogowski coils was as challenging. The continuous Rogowski coils are made of roughly $1 \mathrm{~m}$ long connected segments which often have to step over cooling pipes at the connections. The coils are wound onto SS rods which are individually bent according to the shape of the W7-X plasma vessel. Two groups of 5 rods are connected in series and individually connected to the outside, where they can be used separately in case of a failure in one group rather than being connected in series, which will normally be the case. The Rogowski coils are placed at the same location on the inside as well as the outside of the plasma vessel so that the currents in the vessel can be determined. Again, the wires on the rods need to be well shielded from the ECRH stray radiation. In this case, in order not to compromise the response time of the coils, instead of $\mathrm{Cu}$, thin SS tubes, perforated with $0.7 \mathrm{~mm}$ holes for outgassing, were put over each rod individually and tightly fixed to connection boxes at either end within which their wires are crimped to put them in series. As discussed above, due to the 


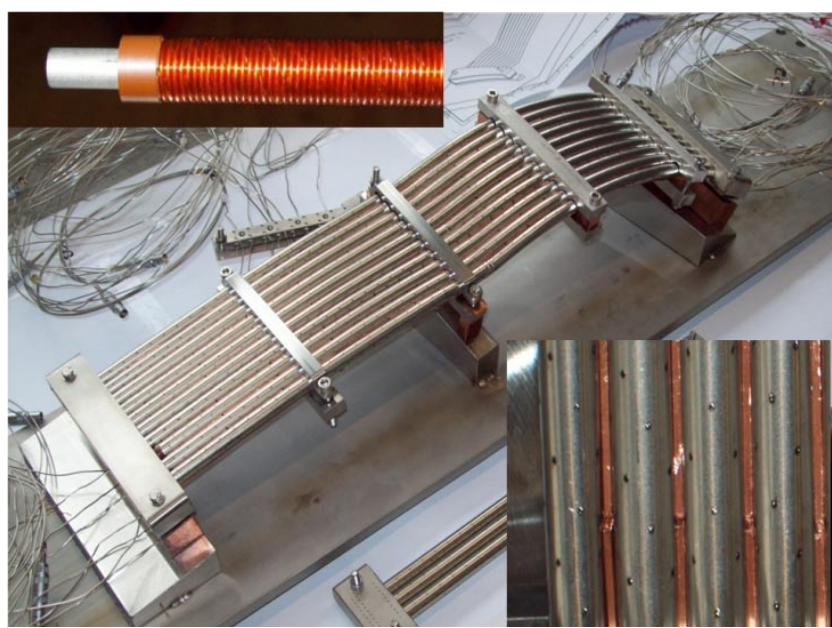

FIG. 3. Mock-up Rogowski coil section (centre) consists of 10 individually 3D shaped rods (top left) with tightly wound wire placed into thin SS tubes for ECRH protection with $0.7 \mathrm{~mm}$ holes for outgassing and copper rails clamped between rods (bottom right) to improve cooling

stray radiation the SS tubes would heat up to unacceptably high temperatures for the wire inside. This made it necessary to tightly clamp between the tubes solid $\mathrm{Cu}$ rails which transport the heat to $\mathrm{Cu}$ blocks which are thermally well connected to the plasma vessel with a Sigraflex ${ }^{\circledR}$ interlayer. In order to achieve the required good thermal contact the plasma vessel is being scanned at those locations to determine its 'as built' geometry and the connecting blocks are machined accordingly (Fig. 3). Pressure sensitive foils were used to prove that the required contact surface area can actually be achieved.

In case of the Mirnov coils, which need to be located close to the plasma just behind small gaps between the carbon tiled plasma vessel wall protection elements, a conducting ECRH shielding would compromise the high frequency response. Therefore, the insulating winding bodies are made of AlN. ${ }^{20}$ This material strongly absorbs the ECRH stray radiation, but due to its excellent heat conductivity and by carefully designing the thermal connection to the wall protection cooling structure it was still possible to keep the body temperatures below $500^{\circ} \mathrm{C}$.

The bolometer cameras are a further diagnostic which is particularly sensitive to ECRH stray radiation. In the first operation phase of W7-X two of the five ports in the triangular plane of the plasma vessel are equipped with a 32 and a 40 channel pinhole camera, providing two fans of near the plasma centre about $5 \mathrm{~cm}$ diameter viewing lines for tomographic reconstruction of the plasma radiation profiles. The gold-foils of the ASDEX-type bolometer detectors have an ECRH absorption coefficient of $0.1 \%$. To ensure that the contribution from ECRH stray radiation becomes negligible, a mesh has been placed just in front of the detectors, which is thermally connected to a surrounding cooling water pipe. With the mesh alone the ECRH induced signal was reduced by a factor 30. By additionally coating the inside of the camera head cavity between the detector and the water cooled plasma facing front plate with a $150 \mu \mathrm{m}$ $\mathrm{Al}_{2} \mathrm{O}_{3} / \mathrm{TiO}_{2}$ absorber layer, a reduction of the stray radiation signal by a further factor 10 was achieved. This in total factor 300 reduction of the stray radiation contribution will be sufficient for the stray radiation signal to be negligible even in the case of low emissive plasmas. ${ }^{21}$

\section{MAINTAINING OPTICAL PROPERTIES}

It is extremely difficult to maintain a calibrated, high optical throughput for diagnostics installed in quasi-stationary operating nuclear fusion devices. Any first optical components that need to be located close to the plasma suffer from reflectivity or transmission losses due to erosion and/or material deposition. If the placement of a mirror in an erosion dominated area cannot be avoided, the only measure that can be taken is to use a special window material which is as much as possible resilient against erosion, like e.g. Molybdenum. For the majority of diagnostics gradual throughput losses caused by material deposition cannot be avoided entirely. However, a number of measures can be taken to maintain the calibration as long as possible, to mitigate any deposition and to clean the surfaces, whenever necessary, from the deposits that could not be avoided.

To maintain the calibration at all times, continuous or frequent measurements of the throughput losses, mostly inflicted on the first plasma facing optical element, need to be performed, either by measuring the deposited layers directly, e.g. with a white light interferometry system, ${ }^{22,23}$ or by in-situ re-calibration or at least relative calibration of the optical systems.

The build-up of mainly hydrogen rich amorphous soft hydrocarbon (a-C:H) layers in W7-X, is tried to be kept at a minimum by in most cases only observing the plasma through small (5 to $10 \mathrm{~mm}$ diameter) pinholes which form the entrance pupil of the special diagnostic optical design. Observing the plasma only through a small pinhole not only reduces the power load on the first optical component significantly but also allows the use of a small uncooled shutter behind the water cooled SS plasma facing front-plate with the pinhole. The shutter will not only be closed during machine conditioning (Helium glow discharge cleaning and boronisation) but also between discharges, since experiments at TEXTOR suggest that most of the carbon layers are deposited between plasma discharges. ${ }^{24}$ Moreover, in the pinhole based systems the efficiency in reducing the build-up of coatings by maintaining a small hydrogen gas flow throughout the discharges will be explored as a further promising measure. ${ }^{25}$ Installing this gas flow system in 9 identical video diagnostic systems will give us the possibility to investigate what is the minimum required flow that still has a worthwhile effect while not affecting general plasma fuelling. In case of diagnostics requiring very high optical throughput and thus large windows, like e.g. the W7-X Thomson scattering or Charge Exchange Recombination Spectroscopy System (CXRS), large actively cooled shutters have been developed ${ }^{14}$ for the same purpose but also to have the possibility to close them in case the diagnostics are not essential for the experimental program (thereby reducing the build-up of coatings) or should the window temperatures unexpectedly reach values higher than their design specification. Furthermore, as learned from the experiments on DIII-D, keeping the first optical element at elevated temperatures $\left(\sim 150^{\circ} \mathrm{C}\right)$ may also prolong the period over which the optical system throughput can be maintained in an acceptable range. ${ }^{26}$

In the end, since already less than a handful of $30 \mathrm{~min}$ plasma discharges would, without any special measures taken, result in a first optical element contamination comparable to the one found after one year of operation in short pulse devices like JET, it will be unavoidable to frequently attempt in-situ cleaning of these components. With laser evaporation techniques and gas discharge cleaning not yet being sufficiently developed, only the method of heating the first plasma facing optical components to $300-400^{\circ} \mathrm{C}$ to remove a large fraction of the soft a-C:H layers overnight or over the weekend will be implemented in some of our diagnostics. ${ }^{27}$ The W7-X imaging diagnostics are examples where many of the above mentioned measures are being implemented. The 10 divertor infrared thermography and visible 
endoscope systems ${ }^{13}$ looking at all 10 discrete divertor modules will be the systems which need to ensure the integrity of the actively cooled W7-X divertor, to be installed in 2018. Being a machine safety control system, the shutter of these systems needs to remain open throughout the entire discharge. Therefore, any measures possible to maintain the data quality and reliability of these systems need to be taken. The about $2 \mathrm{~m}$ long endoscopes (Fig. 4) will consist of a water cooled SS plasma facing front plate with a small, approx. $8 \mathrm{~mm}$ diameter pinhole forming the entrance pupil of the optical system. The light will be transferred via a hyperbolic and a planar mirror to a Cassegrain optics located just outside the port. Outside the port, dichroic beam splitters will then distribute the images onto 8-14 $\mu \mathrm{m}$ and 3-5 $\mu \mathrm{m}$ IR cameras as well as a visible light camera equipped with an interference filter changer. A mirror on the rear side of the secondary mirror of the Cassegrain system provides further possibilities to attach an in-vessel illumination system, a fibre observation optics or a laser beam path onto the divertor. The latter system could be used to gain information on the thickness of re-deposited layers on the divertor targets by measuring the temperature decay time following short laser pulses but also to gain in-situ information during plasma pulses on divertor target deposition and fuel retention using Laser Induced Ablation spectroscopy (LIAS), Laser Induced Breakdown Spectroscopy (LIBS) and Laser Induced Desorption Spectroscopy (LIDS). In this endoscope system a small hydrogen gas flow through the observation pinhole will be maintained throughout the plasma discharges to mitigate soft carbon deposition on the front-end mirrors. The uncooled shutter located on the inboard side of the diagnostic will be closed between and during wall conditioning. The shutter is furthermore equipped with a temperature controlled ceramic heater (e.g. Boralectric ${ }^{\circledR}$ ) which will allow frequent recalibration of the complete IR observation systems as well as relative calibrations in the visible when heating up the ceramic plate to temperatures of about $1000^{\circ} \mathrm{C}$ (Fig. 4). The heater will also be used for cleaning the first mirror by heating it up to $300-400^{\circ} \mathrm{C}$ overnight or over weekends.

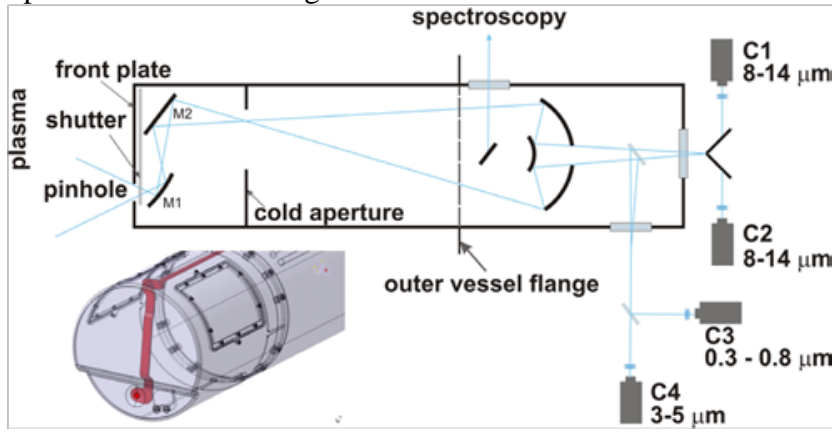

FIG..4. Sketch of the optical layout of the IR/vis. endoscope (C14 cameras); lower inlet shows pinhole closed by shutter (red)

The CMOS cameras for the 10 toroidally viewing video diagnostics $^{28}$ are transported with a special camera carriage through a narrow (100 mm dia.) port which has a bend around a W7-X coil, and then they are locked into a bayonet ring welded into the port. Onto the same ring the vacuum window is mounted from the in-vessel side of the machine. A water cooled pinhole plate, which forms the entrance pupil of the camera lens system, protects the window from plasma radiation heat loads and reduces the ECRH stray radiation penetrating into the system. An ITO coating on the window prevents the ECRH stray radiation from affecting the camera detector. To reduce the ECRH stray radiation inside the cavity formed between the vacuum window and the front plate with the pinhole to a minimum the entire cavity is coated with a $150 \mu \mathrm{m}$ thick ECRH absorbing $\mathrm{Al}_{2} \mathrm{O}_{3} / \mathrm{TiO}_{2}$ layer. Again, a small outflow of hydrogen through the pinhole is being used to reduce window contamination as much as possible. With these diagnostics being located directly above divertor baffles they may be particularly vulnerable to carbon deposition from the divertor. The pinhole will also be closed between plasma discharges and during machine conditioning using a pressure operated Bourdon spring as actuator. $^{14}$

\section{INDIVIDUAL DIAGNOSTIC SPECIFIC ISSUES}

A number of diagnostics have very diagnostic specific long pulse issues that need to be dealt with. We discuss two examples.

In case of the magnetic diagnostics the development of a stable long pulse integrator for the Rogowski coils with its drift being smaller than the lowest expected plasma currents over the entire maximum discharge length of $1800 \mathrm{~s}$ in W7-X was essential. In the system developed, the drift and common mode signals were largely eliminated by chopping the input signal and numerical processing. ${ }^{29}$ The finally achieved drift was measured using an actual plasma vessel sector before it was welded together with other sections and before the W7-X coils were threaded onto the plasma vessel sections. At this very early stage of the W7-X assembly a Rogowski coil had been mounted on the cryo-vacuum side onto a vessel section as a pre-assembly test. By putting a $100 \mathrm{~A}$ wire through this sector an integrator drift of 72 A over $1800 \mathrm{~s}$ had been measured which is very much lower than the lowest expected plasma currents of about $5 \mathrm{kA}$ in W7-X and thus fully sufficient for our purposes. This integrator is also now being tested on $\mathrm{KSTAR}^{30}$ and its basic design has meanwhile been integrated in the present design solution for ITER.

To maintain density control, interferometry, with its capability to measure line of sight averaged densities, is one of the main W7-X control diagnostics for plasma operation. However, nearly all interferometry systems are known to be sensitive to vibrations and to suffer from occasional fringe jumps during rapid density changes. On W7-X it had never been foreseen to build a large rigid and well mechanically damped frame as is used for many interferometer set-ups on present day devices but rather develop an infrared two color interferometer using a $10.6 \mu \mathrm{m} \mathrm{CO}_{2}$ - and $5.3 \mu \mathrm{m}$ CO-laser. It is assumed that the particular feature of this system, of allowing to determine and subtract any signal contributions from vibrations and other thermally induced path length changes, is sufficient to provide a robust line averaged density signal, even with the lasers and retro-reflector mounted to independent, only moderately damped structures. Good progress had already been made with the development of this system ${ }^{7}$ for $\mathrm{W} 7-\mathrm{X}$ when first impressive measurements on TEXTOR with a dispersion interferometer developed by the Budker Institute in Novosibirsk, Russia, demonstrated that this type of interferometer did not even during disruptions suffer from fringe jumps. ${ }^{31}$ The beauty of this system is that in the measured signal any effects from retro-reflector vibrations or thermally induced changes in the laser path length cancel out entirely. This is achieved by creating an additional frequency doubled laser beam from a single, frequency stabilized $\mathrm{CO}_{2}$-laser $(10.6 \mu \mathrm{m})$ by passing it through a frequency doubling

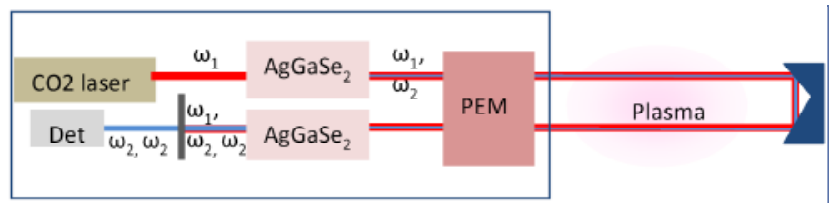

FIG. 5. Principle layout of the W7-X single channel dispersion interferometer with two $\mathrm{AgGaSe}_{2}$ frequency doubling crystals. 
crystal. Thereby two coupled laser beams are passing at two separate wavelengths twice through the plasma, using a retroreflector. Finally the beams again pass through either the same or an additional frequency doubling crystal behind which the primary wavelength is suppressed by a filter. Modulating the phase of the primary laser beam, before it passes through the first frequency doubling crystal, by an electro-optic $(\mathrm{EOM})^{32}$ or photo-elastic modulator (PEM) $)^{33,34}$ one finally creates an interference signal of the remaining $5.3 \mu \mathrm{m}$ beams, of which one passes the plasma at $10.6 \mu \mathrm{m}$ and one at $5.3 \mu \mathrm{m}$, which is then analyzed using lock-in amplifiers (Fig. 5).

The robustness of the interferometer based density control system is for quasi-continuously operating devices of utmost importance. On W7-X the single channel dispersion interferometer system is particularly optimized in this respect. ${ }^{35}$ That means the laser, all optical components and the nitrogen atmosphere inside the optics enclosure up to the entrance port of W7-X are carefully temperature stabilized. Furthermore, two separate frequency doubling crystals are used for the outgoing and returning beams, thereby preventing any spurious light from the returning laser beams from getting back into the laser cavity and affecting its stability. Separating them also makes the temperature stabilization of these very small crystals at the high continuous laser powers, required because of the low efficiency for the frequency doubling, somewhat easier. Similarly a photoelastic modulator $(50 \mathrm{kHz})$ is used in this system which results in a lower time resolution $(\sim 1 \mathrm{~ms})$. However, differently from EOMs, PEMs being a commercially available and relatively simple technology are regarded as more reliable. The retroreflector of this system is located outside a port opposite to the entrance port, sitting on an active vibration compensated table fixed to the Thomson scattering support structure in the W7-X torus centre. The laser beam of the single channel interferometer runs nearly collinear with the laser of the Thomson scattering diagnostic through the specially aligned $\mathrm{W} 7-\mathrm{X}$ port combination allowing easy cross calibration between the two measurements.

Furthermore, a multi-channel dispersion interferometry system, the position and orientation of the viewing lines of which has been optimized using Bayesian techniques ${ }^{36}$ will be installed in a nearby plane spanned by 3 ports. Initially, here the 4 dispersion interferometer modules from TEXTOR will be installed which will later be complemented by a further 6 . These can then serve as back-up in case of a system failure in the single channel system. This is particularly important since with the magnetic field being up all week, also no access to the torus hall will be permitted throughout the week. In these systems EOMs $(250 \mathrm{kHz})$ are used for higher time resolution $(\sim 4 \mu \mathrm{s})$ and only a single frequency doubling crystal is used for the outgoing as well as the returning laser beam. All retro-reflectors for this system need to be placed in-vessel and had to be integrated into the carbon tiled wall heat shield. They are about $2.5 \mathrm{~cm}$ in diameter and will be fixed slightly retracted into the tiles to prevent significant surface erosion. In order to prevent any surface distortions which would affect the wave front phase structure they had to be made of a material with excellent heat transfer capabilities and very small thermal expansion coefficient, like Mo or W. We have chosen Mo as material for the retro-reflectors which is also a well suited mirror material for use in erosion dominated areas. ${ }^{37}$ Whether in this erosion dominated heat shield area the mirror surfaces will be, due to their retracted position deposition dominated or still erosion dominated needs to be seen. Furthermore, one side requires excellent thermal connection to the cooling structure of the heat shield, with the other ones connected to this one such that the heat mainly flows parallel to their surfaces. By minimizing thermal gradients into the depth of the mirror materials any bending of the surfaces is minimized. The connection of the three other mirrors to the one coupled to the cooling structure results in its corners getting hotter than its centre. To minimize surface distortion the Sigraflex foil used to ensure good thermal contact to the cooling structure will be cut out in the centre to reduce its heat transfer capability there, thereby producing a more homogeneous temperature distribution across the surface and thus smaller deformation. The resulting surface deformation is expected to be below $0.2 \mu \mathrm{m}$, $^{9,38}$

\section{SUMMARY}

It has been shown that building diagnostics for quasicontinuously operating fusion devices, even if one does not have to cope with the even more challenging additional issue of high neutron rates as in ITER, still requires many measures to be taken to finally come up with a design solution which is compatible with the requirements. This is hardly ever possible by starting with a conventional short pulse compatible design which is gradually hardened over the years but rather mostly requires already different solutions to start with.

\section{REFERENCES}

${ }^{1}$ H.-S. Bosch, 23rd Fusion Energy Conference, Rep. of Korea, Daejon, 11-16 October 2010, EXC/2-5Rb

${ }^{2}$ V. Erckmann, et al., Fusion Science Technology 52 (2007), 291-312

${ }^{3}$ B. Heinemann, Fusion Engineering 2005, Twenty-First IEEE/NPS Symposium, DOI: 10.1109/FUSION.2005.252886, 2005

${ }^{4}$ J. Boscary, Nucl. Fusion 43, 831 (2003)

${ }^{5}$ H-J Hartfuß, R. König and A.Werner, Plasma Phys. Control. Fusion 48, R83 (2006)

${ }^{6}$ R. Burhenn et al., Contrib. Plasma Phys. 51, 271 (2011)

${ }^{7}$ R. König, et al., Rev. Sci. Instrum. 81, 10E133 (2010)

${ }^{8}$ S. Benhard et al., Fusion Eng. and Design 75463 (2005)

${ }^{9}$ M.Y. Ye, et al., Fus. Eng. and Design 84, 2002 (2009)

${ }^{10} \mathrm{H}$. Thomsen, et al., AIP Conf. Proc. 993, 163 (2008)

${ }^{11}$ R. König, et al., Rev. Sci. Instrum. 75, 4258 (2004)

${ }^{12} \mathrm{~T}$. Eich and A. Werner, Fusion Sci. Technol. 53, 761 (2008)

${ }^{13}$ J. Cantarini et al., Rev. Sci. Instrum. 79, 10F513 (2008)

${ }^{14}$ R. König, et al., Rev. Sci. Instrum. 79, 10-F337 (2008)

${ }^{15}$ O.V. Ogorodnikova et al., J. Nucl. Mater. 341, 175 (2005)

${ }^{16} \mathrm{H}$. Greuner et al., J. Nucl. Mater. 329, 849 (2004)

${ }^{17}$ M. Floristan, et al., Fus. Eng. Des. 86, 1847 (2011)

${ }^{18}$ C. Winnewisser, F. Lewen, H. Helm, Appl. Phys. A66,593 (1998)

${ }^{19}$ J. Ray et al., J.Appl. Phys. 54, 3497 (1983)

${ }^{20}$ U. Neuner et al., Fus. Eng. Des. (2011) DOI:

10.1016/j.fusengdes.2011.11.004

${ }^{21}$ D. Zhang et al., Rev. Sci. Instrum. 81, 10E134 (2010)

${ }^{22}$ F. Hirth et al., Optical Measurement Systems for Industrial Inspection VI, Proc. of SPIE Vol. 7389 73891K-2 (2009)

${ }^{23}$ S.D. Ventura et al., J. Appl. Phys. 97, 043512-12 (2005)

${ }^{24}$ A. Keudell et al., Nucl. Fusion 39, 1451 (1999)

${ }^{25}$ E.E: Mukhin, et al., Nucl. Fus. 52, 013017 (2012)

${ }^{26}$ D.L. Rudakov et al., Phys. Scr. T138, 014007 (2009)

${ }^{27}$ K.Maruyama et al., J. Nucl. Mater. 264, 56 (1999)

${ }^{28}$ J. Sarközi et al., AIP Conf. Proc. 993, 175 (2008)

${ }^{29}$ A. Werner et al., Rev. Sci. Instrum. 79, $10 \mathrm{~F} 122$ (2008)

${ }^{30}$ S.-H. Seon et al., Rev. Sci. Instrum. 81, 123507 (2010)

${ }^{31}$ H. Dreier et al., Rev. Sci. Instrum. 82, 063509 (2011)

${ }^{32}$ P.A. Bagryansky et al., Rev. Sci. Instrum. 77, 053501 (2006)

${ }^{33}$ T. Akiyama et al., Rev. Sci. Instrum. 81, 10D501 (2010)

${ }^{34}$ T. Akiyama et al., Plasma and Fusion Research: Rapid Communications Vol. 5, 047 (2010)

${ }^{35} \mathrm{P}$. Kornejew, to be published EPS 2012

${ }^{36}$ H. Dreier et al., Rev. Sci. Instrum. 79, 10E712 (2008)

${ }^{37}$ A. Litnovsky et al., Nucl. Fusion 47, 833 (2007)

${ }^{38}$ M. Köppen et al., Fus. Eng. \& Design 86, 11166 (2011) 